\title{
Reducción de tiempos de espera en el cambio de modelo mediante la aplicación de la herramienta SMED, un caso de estudio
}

\author{
Reduction of waiting times at the change of model through the application of SMED \\ tool, a case study
}

\author{
MARTINEZ-HERNANDEZ, Julio Cesarł*, CRUZ-SOLIS, Edgar Jesús, GARRIDO-ROSADO, Rafael \\ y SANTIAGO-ESCUDERO, Anselmo
}

Instituto Tecnológico Superior de Huauchinango / Instituto Tecnológico Superior de la Sierra Norte de Puebla

ID 1er Autor: Julio Cesar, Martínez-Hernández / ORC ID: 0000-0001-9528-156X, Researcher ID Thomson: I-3507-2018 arXiv Author ID: 0000-0001-9528-156X, CVU CONACYT ID: 904537

ID 1er Coautor: Edgar Jesús, Cruz-Solís / ORC ID: 0000-0003-4083-0888, Researcher ID Thomson: I-4334-2018, arXiv Author ID: 0000-0003-4083-0888, CVU CONACYT ID: 904718

ID 2do Coautor: Rafael, Garrido-Rosado / ORC ID: 0000-0002-7703-5450, Researcher ID Thomson: D-4420-2019, arXiv Author ID: 0000-0002-7703-5450, CVU CONACYT ID: 639139

ID 3er Coautor: Anselmo, Santiago-Escudero / ORC ID: 0000-0003-3409-3826, Researcher ID Thomson: F-5198-2019 arXiv Author ID: 0000-0003-3409-3826, CVU CONACYT ID: 971224

DOI: $10.35429 /$ JIE.2019.8.3.21.29

Recibido 24 de Abril, 2019, Aceptado, 19 de Junio, 2019

\section{Resumen}

En gestión de la producción, SMED (acrónimo de SingleMinute Exchange of Die) derivada de la metodología Lean Manufacturing, se puede interpretar como una filosofía de trabajo nacida en Toyota, su aplicación está basada en la eliminación del desperdicio de forma sostenible en el tiempo, permitiendo mejorar la productividad. Esta investigación presenta la aplicación de la metodología SMED en una empresa especializada en la producción de embalaje del tipo Honeycomb, donde la problemática principal se ubicaba en la línea de CHS, esta produce el componente principal del producto, sin embargo, el tiempo de demora es de hasta 71 minutos por cambio de modelo, ajuste de la máquina y posteriormente el arranque, reflejándose en 1500 libras de producto retrasado, viéndose afectado en los tiempos de respuesta al cliente, generándose horas extraordinarias e incumpliendo con las metas de producción diarias. El objetivo de esta investigación es reducir el tiempo de preparación y montaje de los herramentales de acuerdo con los cambios de modelo solicitados, a través de la aplicación del SMED se obtendrá un sistema de producción flexible que pueda responder a los constantes cambios en el mercado, reducción de tiempos de entrega y aumento en la capacidad de producción.

\begin{abstract}
In the management of the production, SMED (acronym of Single-Minute Exchange of Die) derived from the Lean Manufacturing methodology can be interpreted as a philosophy of work born in Toyota, its application is based on the elimination of waste in a sustainable way over time, allowing improve the productivity. This research presents the application of the SMED methodology in a company specialized in the production of honeycomb type packaging, where the main problem is in the line of $\mathrm{CHS}$, this produces the main component of the product, however, the delay time is up to 71 minutes per model change, adjustment of the machine and then the start, reflecting in 1500 pounds of delayed product, being affected in customer response times, generating overtime and not meeting the daily production goals. The objective of this research is to reduce the time of preparation and assembly of the tooling according to the requested changes, through the application of SMED a flexible production system will be obtained that allows responding to the constant changes in the market, reduction of delivery times and increase in production capacity.
\end{abstract}

Producción, Sistema, Reducción

Production, System, Reduction

Citación: MARTINEZ-HERNANDEZ, Julio Cesar, CRUZ-SOLIS, Edgar Jesús, GARRIDO-ROSADO, Rafael y SANTIAGO-ESCUDERO, Anselmo. Reducción de tiempos de espera en el cambio de modelo mediante la aplicación de la herramienta SMED, un caso de estudio. Revista de Ingeniería Industrial. 2019 3-8: 21-29

\footnotetext{
* Correspondencia del Autor (Correo electrónico: jc.martinez@ @uauchinango.tecnm.mx)

$\dagger$ Investigador contribuyendo como primer autor. 


\section{Introducción}

La manufactura esbelta reúne diversos métodos y técnicas que son desarrollados a lo largo del sistema de producción de la empresa, enfocándose en la reducción o eliminación de desperdicios.

Existen distintas definiciones de desperdicio de acuerdo con cada autor o enfoque que se le dé a la palabra, según la Real Academia Española (2018) define al desperdicio como: "residuo de lo que no se puede o no es fácil de aprovechar o se deja de utilizar por descuido".

Hablar de desperdicio en producción es referirse a todas las actividades que no agregan valor al producto o servicio que se le brinda al cliente. Socconini (2008) menciona que en muchos de los casos sólo del 5\% al 10\% de todas las actividades desarrolladas en las empresas agregan valor y lo restante es desperdicio.

En la actualidad las empresas deben de tomar conciencia de los desperdicios generados dentro de sus procesos de producción, especialmente el tiempo perdido, a fin de reducirlo al máximo y ser más productivos.

El impacto de los desperdicios es la razón principal de una baja competitividad de las empresas ante el mercado, debido a que no se trabaja bajo un enfoque esbelto, ocasionando el incumplimiento de los requisitos de sus clientes.

Roqueme y Suarez (2015) señalan que para reducir al máximo los desperdicios, muchas empresas en la actualidad optan por las herramientas del principio Lean Manufacturing que suponen un enfoque de mejora continua para alcanzar una mayor productividad y éxito empresarial.

La preparación rápida es una innovación aportada por los japoneses en la organización científica del trabajo. Efectivamente, el sistema SMED, según su creador Shigeo Shingo, tiene sus orígenes en ciertos trabajos que le fueron encargados, en 1950, en la fábrica Toyo Kogyo de Mazda. Sin embargo, se desarrolló completamente alrededor de los años setenta del siglo pasado cuando realizaba trabajos para Toyota y ésta adoptó, promovida por los propios operarios, el sistema SMED como uno de los pilares básicos de su modo de fabricación (Rajadell, 2010).
Los tiempos de espera son el desperdicio más común dentro de las empresas, por ejemplo; esperar a que la máquina termine su ciclo de producción o cuando un operario espera a otro para poder empezar o finalizar una actividad, así como el tiempo que se necesita para el cambio de un producto o modelo y la preparación de una máquina.

La diversificación de la demanda ha exigido una rápida adaptación de los sistemas de producción, en particular para los sistemas de producción que trabajan bajo pedido en los que se requiere controlar tanto la cantidad de cambios de referencia como desarrollar actividades muy ajustadas para reducir el tiempo de preparación de sus equipos y generar eficiencia lo que redunda en la disminución de los tiempos de entrega, considerado hoy como elemento esencial en la calidad del servicio (Vergara, 2019).

Lo anterior sirve de base para comprender la oportunidad de mejora que se presenta en el caso de estudio realizado en una empresa productora de embalaje de cartón secundario. La empresa cuenta con 5 líneas de producción comenzando con CHS, PANEL, CONVERTION, MABE'S CORNER BOARD Y CORNER BOARD.

La línea de producción CHS es considerada como el motor de la empresa ya que produce el componente principal del producto, la producción es medida en libras, el tiempo de ciclo depende del modelo y espesor que se fabrique. Sin embargo, los paros en esta línea de producción son originados por la preparación de herramientas y cambios de modelo, generando tiempos de espera de por lo menos 60 minutos, tiempo que representa el atraso mayor a 1,500 libras, evidenciando una baja productividad e incumplimientos en las metas de producción diarias.

El objetivo del proyecto es reducir el tiempo de preparación y montaje de los herramentales por cambio de modelo, con la finalidad de incrementar la productividad en la línea CHS en una empresa productora de embalaje tipo Honeycomb. El proyecto contribuye sustancialmente a la aplicación de conocimientos teóricos llevados a la práctica, a través de la adquisición de experiencia para el equipo de trabajo y el impacto beneficio para la empresa.

MARTINEZ-HERNANDEZ, Julio Cesar, CRUZ-SOLIS, Edgar Jesús, GARRIDO-ROSADO, Rafael y SANTIAGO-ESCUDERO, Anselmo. Reducción de tiempos de espera en el cambio de modelo mediante la aplicación de la herramienta SMED, un caso de estudio. Revista de Ingeniería Industrial. 2019 


\section{Metodología}

De acuerdo con la clasificación de Gordon Dankhe (1986), las investigaciones se dividen en: exploratorias, descriptivas, correlaciónales y explicativas. Los estudios descriptivos por lo general son la base de las investigaciones correlacionales, las cuales a su vez proporcionan información para llevar a cabo estudios explicativos que generan un sentido de entendimiento y son altamente estructurados. (Hernández, 2010).

El proyecto pretende reducir el tiempo de preparación, de modo que se propone realizar un estudio de alcance descriptivo correlacional, el cual nos brinda la posibilidad de describir como el método actual tiene efectos negativos en la productividad de la línea CHS. Para el desarrollo de esta investigación se efectuará un diseño experimental de tipo cuasiexperimental, descriptivo correlacional, ya que se interactúa con la metodología SMED (variable independiente), observando los efectos sobre la productividad (variable dependiente). Por su parte Valderrama (2013), sugiere manipular la variable independiente para ver los efectos que se crean en la variable dependiente.

En la figura 1, se describen las etapas desarrolladas para lograr el objetivo del proyecto, estas fueron el resultado de una extensa revisión bibliográfica combinada con las necesidades de este caso de estudio.

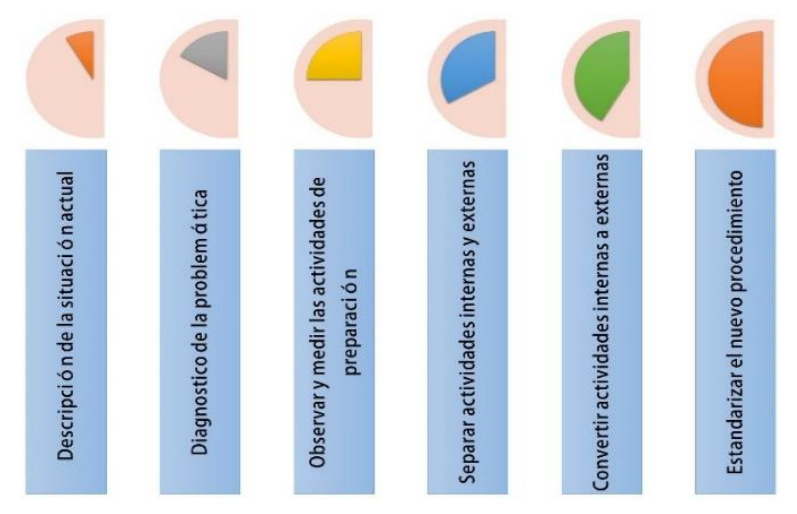

Figura 1 Metodología propuesta Fuente: Elaboración Propia

\section{Descripción de la situación actual}

La empresa cuenta con tres turnos con jornadas laborales de 12 horas, con 25 personas interactuando en el proceso, distribuidas en las áreas de producción en su mayoría, almacén y embarques, excluyendo al personal directivo.
Existen 5 líneas de producción, en las cuales se comienza con la elaboración de Core (material de soporte para el producto con forma de nido de abeja) en distintos espesores, éste es utilizado para la fabricación de paneles que pueden ser para venta directa o bien realizarle transformaciones en las siguientes estaciones, obteniendo como resultados separadores, protectores de esquina, etc.

En la figura 2 se muestra la maquina CHS (Core Machine Sections), esta integra la línea principal de producción, es operada por dos personas (líder del área y asistente), requiere de 6 rollos de papel de un peso aproximado de 4,100 libras, no obstante, puede operar con 4 .

Los 6 rollos son colocados en porta rollos rotativos, cada papel es desenvuelto y colocado a través de un manifold que le aplica resistol en líneas para después ser empalmado con los demás papeles, éstos se dirigen de manera horizontal a una cuchilla para cortarlos en tiras que caen verticalmente en una mesa pegándose uno con otro convirtiéndose en Core. El tiempo de ciclo depende del espesor que se realice y de los cortes por minutos configurados, siendo el estándar de 550 CPM.

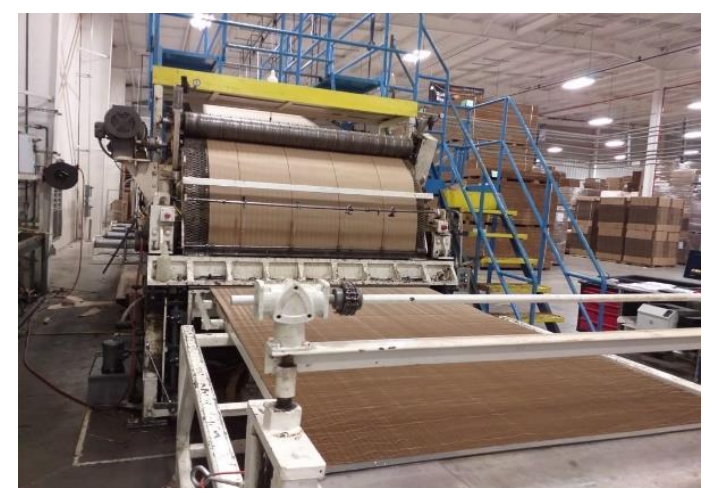

Figura 2 Core Machine Sections

Las actividades que se realizan en la línea CHS son: el estibado del producto, preparación de la máquina y herramienta (cambio de rollo, cambio de modelo), la colocación o configuración de manifolds, el jalado y corte de papel para su alineación, el ajuste del espesor a manufacturar, entre otras.

El tiempo de espera (paro de la línea) va de por lo menos 60 minutos, siendo una actividad critica dentro de la operación diaria todas las tareas relacionadas con el ajuste por cambio de modelo, preparación de herramientas y arranque de máquina.

MARTINEZ-HERNANDEZ, Julio Cesar, CRUZ-SOLIS, Edgar Jesús, GARRIDO-ROSADO, Rafael y SANTIAGO-ESCUDERO, Anselmo. Reducción de tiempos de espera en el cambio de modelo mediante la aplicación de la herramienta SMED, un caso de estudio. Revista de Ingeniería Industrial. 2019 
El tiempo no aprovechado representa el $24 \%$ del total de la jornada laboral, causando grandes retrasos en el programa de producción diario, sin considerar los paros de línea por mantenimiento preventivo y correctivo, esto fomenta un sistema de producción débil e incrementa las operaciones que no agregan valor al producto, sin mencionar el desabasto de materia prima para el proceso de manufactura de las siguientes líneas.

\section{Diagnóstico de la problemática}

Para poder determinar los problemas significativos que afectan la productividad de la línea CHS, se emplean herramientas administrativas (lluvia de ideas) y estadísticas (diagrama de Ishikawa y diagrama de Pareto). En la figura 3 se muestra el diagrama de causaefecto o de Ishikawa, el cual es un método gráfico que relaciona un problema o efecto con los factores o causas que posiblemente lo generan.

La importancia de este diagrama radica en que obliga a buscar las diferentes causas que afectan el problema bajo análisis $\mathrm{y}$, de esta forma, se evita el error de buscar de manera directa las soluciones sin cuestionar cuáles son las verdaderas causas (Gutierrez,2009).

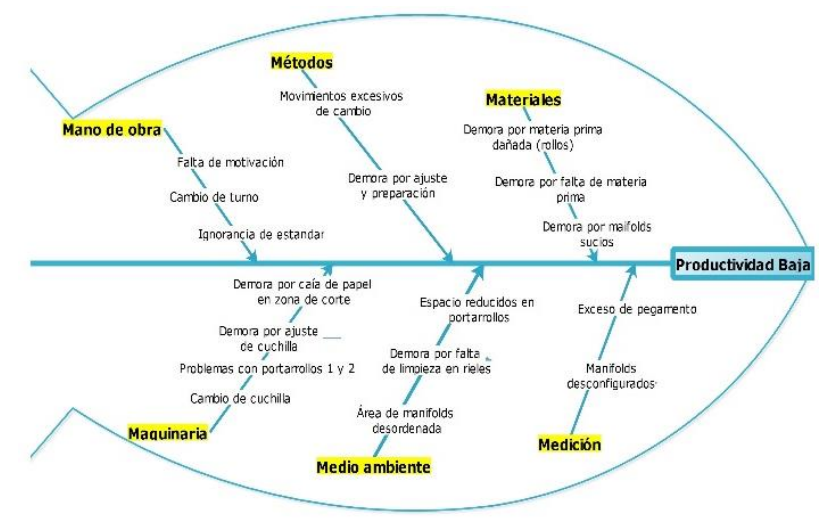

Figura 3 Diagrama de Ishikawa productividad baja

No se pretende resolver todos los problemas de un proceso o atacar todas las causas al mismo tiempo. En este sentido, el diagrama de Pareto es un gráfico especial de barras cuyo campo de análisis son los datos categóricos y ayudar a localizar los problemas vitales, así como sus causas más importantes (Gutiérrez, 2010). En el gráfico 1, se muestra la incidencia de los problemas considerados y sus frecuencias presentadas durante el periodo de observación de 30 días en la línea CHS.

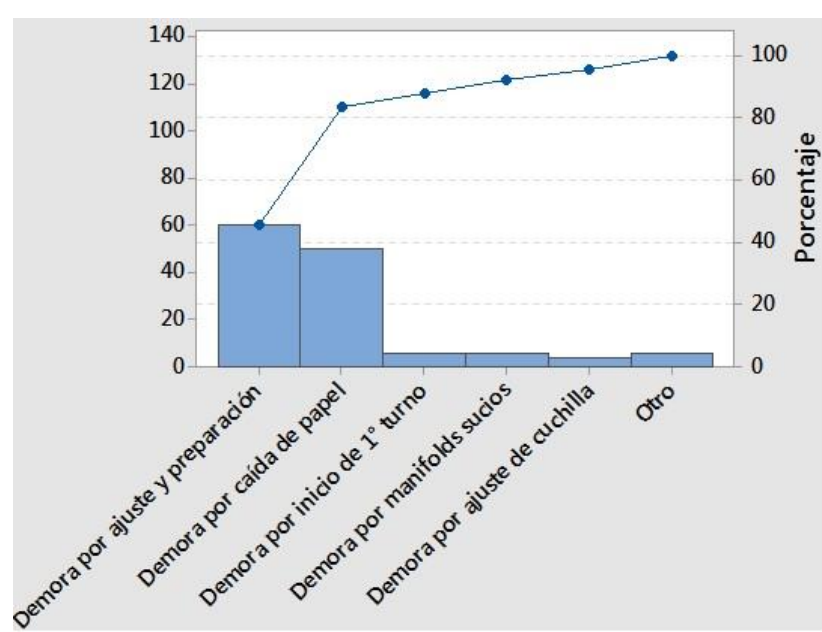

Gráfico 1 Diagrama de Pareto tiempos de espera Fuente: Elaboración Propia

Se observa en la tabla 1 que los principales problemas de retraso que influyen en la productividad son los tiempos de espera causados por el ajuste, preparación y cambio de modelo con $45.5 \%$ de incidencia, seguido de la caída de papel en zona de ajuste con $37.9 \%$.

De acuerdo con los porcentajes presentados se determinó reducir el tiempo por ajuste, preparación y cambio de modelo, con la finalidad de mejorar la productividad en la línea CHS.

\begin{tabular}{|l|r|r|r|}
\hline \multicolumn{1}{|c}{$\begin{array}{c}\text { Problemas } \\
\text { registrados }\end{array}$} & Frecuencia & Porcentaje & $\begin{array}{r}\text { Porcentaje } \\
\text { acumulado }\end{array}$ \\
\hline $\begin{array}{l}\text { Demora por ajustes } \\
\text { y preparación }\end{array}$ & 60 & 45.5 & 45.5 \\
\hline $\begin{array}{l}\text { Demora por caída } \\
\text { de papel en zona de } \\
\text { corte }\end{array}$ & 50 & 37.9 & 83.3 \\
\hline $\begin{array}{l}\text { Demora por inicio } \\
\text { de 1 turno (Lunes) }\end{array}$ & 6 & 4.5 & 87.9 \\
\hline $\begin{array}{l}\text { Demora por } \\
\text { manifolds sucios }\end{array}$ & 6 & 4.5 & 92.4 \\
\hline $\begin{array}{l}\text { Demora por ajuste } \\
\text { de cuchilla }\end{array}$ & 4 & 3.0 & 95.5 \\
\hline Otro & 6 & 4.5 & 100.0 \\
\hline
\end{tabular}

Tabla 1 Porcentajes de los problemas registrados Fuente: Elaboración Propia

\section{Observar y medir las actividades de preparación}

Realizar una preparación para un nuevo modelo en la Línea CHS requiere de un gran número de actividades ya que es conformada de 193, obteniendo la medición se observaron que aquellas de mayor tiempo en minutos fueron desde el abastecimiento de materia prima, montaje de rollos, jalado de papel hasta el ajuste de turca, como se menciona en la tabla 2 donde se registraron las observaciones críticas. 


\begin{tabular}{|l|l|l|l|l|l|l|}
\hline No & \multicolumn{1}{|c}{ Actividades } & \multicolumn{1}{c|}{$\begin{array}{l}\text { Tiempo } \\
(\mathbf{m i n})\end{array}$} & \multicolumn{1}{c|}{ No } & \multicolumn{1}{c|}{ Actividades } & \multicolumn{1}{l|}{$\begin{array}{l}\text { Tiempo } \\
\text { (min) }\end{array}$} \\
\hline 40 & $\begin{array}{l}\text { Entregar } \\
\text { solicitud a } \\
\text { almacén }\end{array}$ & 1.353 & 49 & $\begin{array}{l}\text { Extraer } \\
\text { rollo } \\
\text { sobrante }\end{array}$ & 4.743 \\
\hline 41 & $\begin{array}{l}\text { Regresar a } \\
\text { máquina CHS }\end{array}$ & 1.332 & 51 & $\begin{array}{l}\text { Montar } \\
\text { rollo nuevo }\end{array}$ & 1.684 \\
\hline 42 & $\begin{array}{l}\text { Esperar } \\
\text { abastecimiento } \\
\text { de rollos }\end{array}$ & 18.682 & 162 & Jalar papel & 12.395 \\
\hline 43 & $\begin{array}{l}\text { Buscar } \\
\text { herramientas }\end{array}$ & 1.523 & 188 & $\begin{array}{l}\text { Ajustar } \\
\text { tuerca }\end{array}$ & 1.166 \\
\hline 45 & Retirar núcleos & 1.858 & & & \\
\hline
\end{tabular}

Tabla 2 Medición de tiempos de cambio Fuente: Elaboración Propia

En la figura 4 se muestran las actividades básicas de preparación y ajuste para cambio de modelo, iniciando con la solicitud de rollos y terminando con el ajuste del modelo a manufacturar.

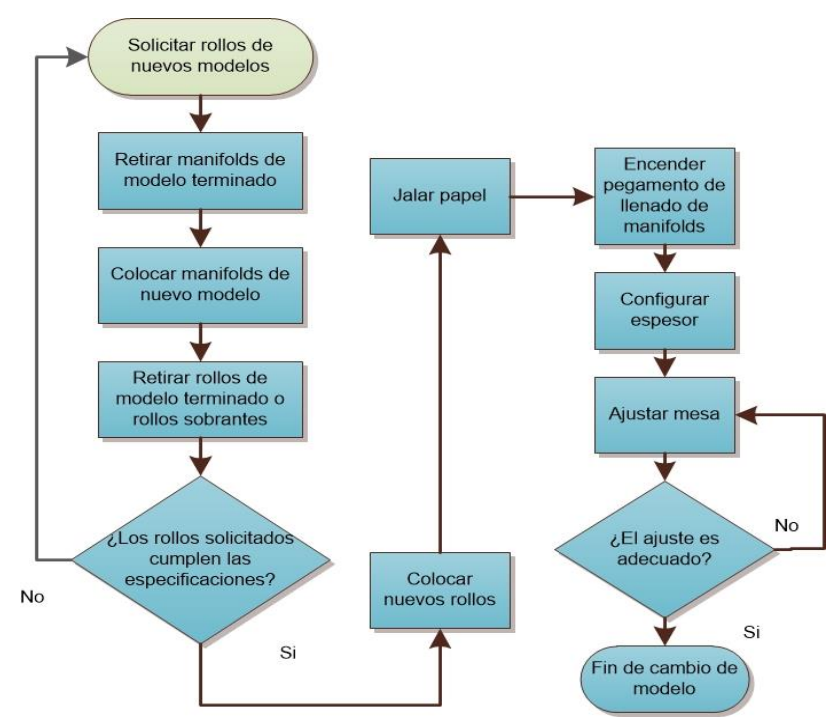

Figura 4 Diagrama de Flujo preparación, ajuste para cambio de modelo en línea CHS

Fuente: Elaboración Propia

En la figura 5, podemos observar que se tiene un descontrol con los manifolds en cuanto a orden y limpieza, por lo tanto, se demora en su búsqueda y colocación de estos, afectando el tiempo de cambio en los modelos.

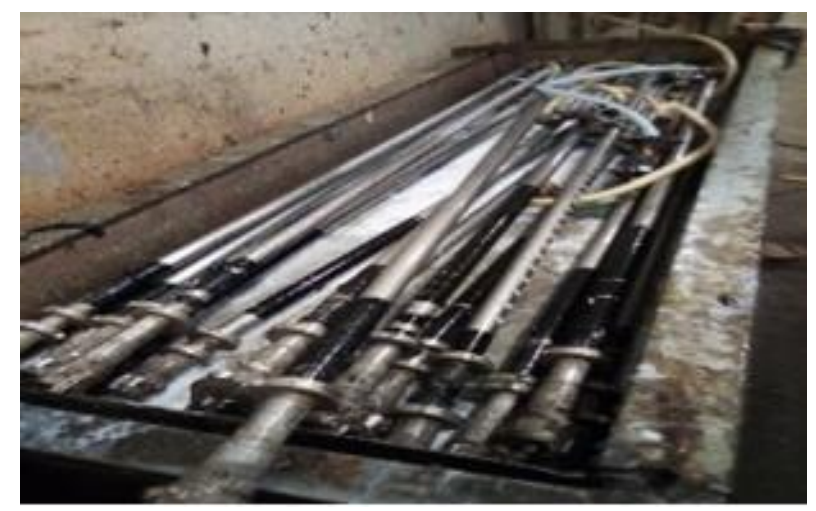

Figura 5 Área de manifolds

Fuente: Empresa (caso de estudio)
En esta fase se procedió a realizar un diagrama de flujo de proceso para visualizar cada una de las etapas necesarias para realizar un ajuste, preparación y cambio de modelo en la línea CHS, en la tabla 3 se muestra un resumen de los eventos registrados en el diagrama con la finalidad de identificar áreas de oportunidad.

\begin{tabular}{|l|r|}
\hline \multicolumn{1}{|c|}{ Evento } & Presente \\
\hline Operación & 103 \\
\hline Transporte & 84 \\
\hline Retrasos & 4 \\
\hline Inspección & 2 \\
\hline Almacenamiento & 0 \\
\hline
\end{tabular}

Tabla 3 Resumen de eventos diagrama de flujo de proceso Fuente: Elaboración Propia

\section{Separación de actividades internas y externas}

De las 193 actividades registradas para el cambio de modelo se procede a la separación de actividades externas de las internas, por lo que se logran identificar 87, en la tabla 4 , se indican aquellas actividades críticas que pueden realizarse con la máquina encendida.

\begin{tabular}{|c|l|c|c|l|c|}
\hline No & \multicolumn{1}{c}{ Actividades } & \multicolumn{1}{c|}{$\begin{array}{c}\text { Tiempo } \\
\text { (min) }\end{array}$} & No & Actividades & $\begin{array}{c}\text { Tiempo } \\
\text { (min) }\end{array}$ \\
\hline 40 & $\begin{array}{l}\text { Entregar } \\
\text { solicitud de } \\
\text { rollo a almacén }\end{array}$ & 1.353 & 49 & $\begin{array}{l}\text { Extraer rollo } \\
\text { sobrante 1 }\end{array}$ & 4.743 \\
\hline 41 & $\begin{array}{l}\text { Regresar a } \\
\text { máquina CHS }\end{array}$ & 1.332 & 51 & $\begin{array}{l}\text { Montar rollo } \\
\text { nuevo 1 }\end{array}$ & 1.684 \\
\hline 42 & $\begin{array}{l}\text { Esperar } \\
\text { abastecimiento } \\
\text { de rollos }\end{array}$ & 18.682 & 72 & $\begin{array}{l}\text { Extraer rollo } \\
\text { sobrante 2 }\end{array}$ & 4.743 \\
\hline 43 & $\begin{array}{l}\text { Buscar } \\
\text { herramientas }\end{array}$ & 1.523 & 73 & $\begin{array}{l}\text { Montar rollo } \\
\text { nuevo 2 }\end{array}$ & 1.684 \\
\hline 45 & Retirar núcleos & 1.858 & & & \\
\hline
\end{tabular}

Tabla 4 Separación de actividades internas y externas Fuente: Elaboración Propia

\section{Convertir actividades internas en externas}

En la tabla 5, se muestra el extracto de la conversión de actividades internas a externas y su respectivo ahorro en tiempo, dentro de las mejoras aplicadas fue el cambio de 2 de 6 rollos (1 y 2) de papel sin realizar paro de máquina, dar seguimiento a 5's en orden y limpieza para disminuir el tiempo de búsqueda de manifolds y herramental, solicitar materia prima antes del paro, realizar actividades de limpieza e inspección después del arranque. 


\begin{tabular}{|c|c|c|c|c|c|}
\hline No & Actividades & $\begin{array}{l}\text { Tiempo } \\
\text { antes } \\
\text { (min.) }\end{array}$ & $\begin{array}{l}\text { Tiempo } \\
\text { después }\end{array}$ & Interno & Externo \\
\hline 24 & $\begin{array}{l}\text { Buscar nuevos } \\
\text { manifold } 5\end{array}$ & 0.305 & 0 & A. I & A. $\mathrm{E}$ \\
\hline 43 & $\begin{array}{l}\text { Llenar } \\
\text { solicitud de } \\
\text { rollos }\end{array}$ & 0.851 & 0 & A. I & A. $\mathrm{E}$ \\
\hline 44 & $\begin{array}{l}\text { Entregarlo a } \\
\text { almacén }\end{array}$ & 1.353 & 0 & A. I & A. $E$ \\
\hline 45 & $\begin{array}{l}\text { Regresar a } \\
\text { máquina } \mathrm{CHS}\end{array}$ & 1.332 & 0 & A. I & A. $E$ \\
\hline 46 & $\begin{array}{l}\text { Esperar } \\
\text { abastecimiento }\end{array}$ & 18.682 & 0 & A. I & A. $E$ \\
\hline 47 & $\begin{array}{l}\text { Buscar } \\
\text { herramientas }\end{array}$ & 1.523 & 0 & A. I & A. $E$ \\
\hline 49 & Retirar núcleos & 1.858 & 0 & A. I & A. E \\
\hline 52 & $\begin{array}{ll}\text { Mover } & \\
\text { portarrollo } & 1 \mathrm{~A} \\
\text { al área } & \text { de } \\
\text { extracción } & \end{array}$ & 1.665 & 0 & A. I & A. $E$ \\
\hline 53 & $\begin{array}{ll}\begin{array}{l}\text { Extraer } \\
\text { sobrante }\end{array} & \text { rollo } \\
\end{array}$ & 4.743 & 0 & A. I & A. $\mathrm{E}$ \\
\hline 55 & $\begin{array}{ll}\begin{array}{l}\text { Montar } \\
\text { nuevo }\end{array} & \text { rollo } \\
\end{array}$ & 1.684 & 0 & A. I & A. $\mathrm{E}$ \\
\hline 72 & $\begin{array}{ll}\text { Mover } & \\
\text { portarrollo } 2 \text { al } \\
\text { área } \\
\text { extracción }\end{array}$ & 1.665 & 0 & A. I & A. $\mathrm{E}$ \\
\hline
\end{tabular}

Tabla 5 Conversión de actividades Internas a Externas

En la tabla 6, se indica el resumen de los eventos registrados después de la optimización de actividades

\begin{tabular}{|l|r|}
\hline \multicolumn{1}{|c|}{ Evento } & Presente \\
\hline Operación & 65 \\
\hline Transporte & 51 \\
\hline Retrasos & 1 \\
\hline Inspección & 0 \\
\hline Almacenamiento & 0 \\
\hline
\end{tabular}

Tabla 6 Resumen de eventos diagrama de flujo de proceso optimizado

\section{Estandarizar el nuevo procedimiento}

Después de realizar observaciones, separaciones, mejoras en actividades convirtiéndolas de internas a externas, se determinó el tiempo estándar promedio para el tiempo operativo de 10.54 horas, esta información se presenta en la tabla 7.

\begin{tabular}{|c|c|c|c|c|c|}
\hline No. & $\begin{array}{c}\text { Tiempo } \\
\text { operativo }\end{array}$ & $\begin{array}{l}\text { No. De } \\
\text { cambios }\end{array}$ & $\begin{array}{c}\text { Tiempo } \\
\text { operativo }\end{array}$ & $\begin{array}{l}\text { No. De } \\
\text { cambios }\end{array}$ & $\begin{array}{c}\text { Tiempo } \\
\text { operativo }\end{array}$ \\
\hline 1 & 10.55 & 21 & 10.55 & 41 & 10.52 \\
\hline 2 & 10.52 & 22 & 10.55 & 42 & 10.56 \\
\hline 3 & 10.55 & 23 & 10.52 & 43 & 10.55 \\
\hline 4 & 10.52 & 24 & 10.53 & 44 & 10.55 \\
\hline 5 & 10.53 & 25 & 10.53 & 45 & 10.52 \\
\hline 6 & 10.51 & 26 & 10.54 & 46 & 10.53 \\
\hline 7 & 10.55 & 27 & 10.54 & 47 & 10.56 \\
\hline 8 & 10.55 & 28 & 10.53 & 48 & 10.55 \\
\hline 9 & 10.55 & 29 & 10.53 & 49 & 10.55 \\
\hline 10 & 10.55 & 30 & 10.52 & 50 & 10.53 \\
\hline \multicolumn{5}{|r|}{ Promedio } & 10.54 \\
\hline
\end{tabular}

Tabla 7 Resumen de eventos diagrama de flujo de proceso optimizado
Dentro de las actividades realizadas en esta etapa se organizó el área de manifolds en dos clasificaciones; los señalados de rojo son de uso frecuente (99\%); mientras que los manifolds señalados con la flecha azul, su uso corresponde al $1 \%$ o menor, esto derivado de operaciones especiales (trabajo con papel reciclado), tal y como se muestra en la figura 6 .

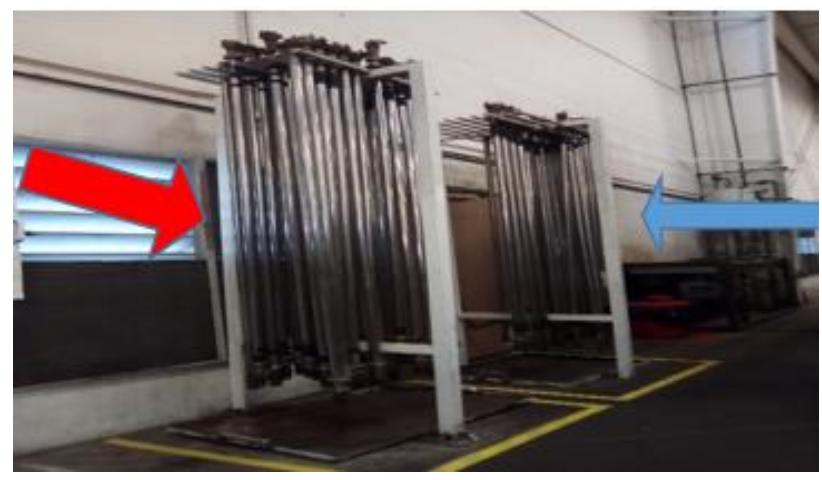

Figura 6 Clasificación de manifolds

Fuente: Empresa (caso de estudio)

Las 5 's son una herramienta esencial para facilitar las actividades de mejora en un cambio de producto (Socconini, 2008), derivado de lo anterior se realizaron diferentes actividades referentes a esta herramienta, se muestran en la figura 7.

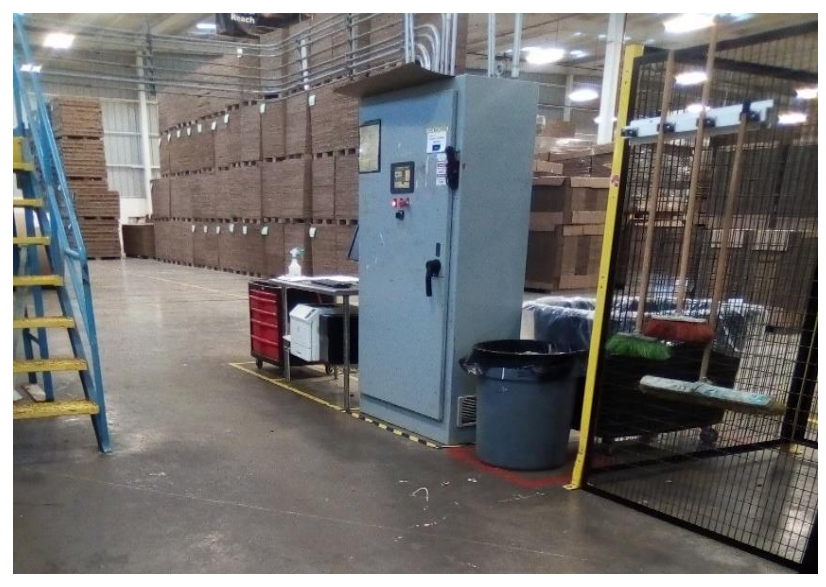

Figura 7 Actividades de 5 's en el área de CHS Fuente: Empresa (caso de estudio)

\section{Resultados}

Para un análisis más detallado en esta etapa se consideraron las dimensiones de tiempo de paro por cambio de modelo (ajuste y preparación) y disponibilidad de la máquina, como parte de la variable independiente; a su vez se consideró a la eficiencia y eficacia como dimensiones para la variable dependiente (productividad), como se puede apreciar en la tabla 8 .

MARTINEZ-HERNANDEZ, Julio Cesar, CRUZ-SOLIS, Edgar Jesús, GARRIDO-ROSADO, Rafael y SANTIAGO-ESCUDERO, Anselmo. Reducción de tiempos de espera en el cambio de modelo mediante la aplicación de la herramienta SMED, un caso de estudio. Revista de Ingeniería Industrial. 2019 


\begin{tabular}{|l|l|l|}
\hline \multicolumn{1}{|c|}{ Variables } & \multicolumn{1}{c|}{ Dimen. } \\
\multirow{4}{*}{$\begin{array}{l}\text { Independiente } \\
\text { SMED }\end{array}$} & $\begin{array}{l}\text { Cambio de } \\
\text { modelo }\end{array}$ & $\begin{array}{l}\text { TCC } \\
\text { Tiempo consumido por cambio } \\
\text { TCC }=\text { Tiempo disponible } \\
\text { cambio }\end{array}$ \\
\cline { 2 - 3 } & $\begin{array}{l}\text { Disponibili } \\
\text { dad }\end{array}$ & $\begin{array}{l}\text { DM }=\frac{\text { Tiempo operativo }}{\text { Tiempo disponible }} \\
\text { DM= Disponibilidad de maquina }\end{array}$ \\
\hline \multirow{2}{*}{$\begin{array}{l}\text { Dependiente } \\
\text { Productividad }\end{array}$} & Eficiencia & $\begin{array}{l}\text { Eficiencia } \\
=\frac{\text { Horas máquinas realizadas }}{\text { Horas máquinas programadas }}\end{array}$ \\
\cline { 2 - 3 } & Eficacia & $\begin{array}{l}\text { Eficacia } \\
=\frac{\text { Producción real }}{\text { Producción programada }}\end{array}$ \\
\hline
\end{tabular}

Tabla 8 Matriz de operacionalización de variables Fuente: Elaboración Propia

En relación con los tiempos consumidos por cambios de modelo, se registraron durante un periodo de 30 días, el tiempo promedio obtenido fue de 70.99 minutos (1.18 horas), considerando 71 minutos.

\begin{tabular}{|c|c|l|l|}
\hline Variable & \multicolumn{2}{c}{ Dimen. } & \multicolumn{2}{c|}{ Fórmula } & \multicolumn{1}{c|}{ Res. } \\
\hline \multirow{3}{*}{ Productividad } & Eficiencia & $\begin{array}{l}\text { Eficiencia } \\
=\frac{\text { Horas máquinas } r}{\text { Horas máquinas prog }}\end{array}$ & $\frac{9.82 \mathrm{hrs}}{11 \mathrm{hrs}}=\mathbf{0 . 8 9}$ \\
\cline { 2 - 4 } & Eficacia & $\begin{array}{l}\text { Eficacia } \\
=\frac{\text { Producción real }}{\text { Producción program }}\end{array}$ & $\frac{12180 \text { libras }}{16000 \text { libras }}=\mathbf{0 . 7 6}$ \\
\hline
\end{tabular}

En el gráfico 2 se muestra el comportamiento de los tiempos por cambio de modelo.

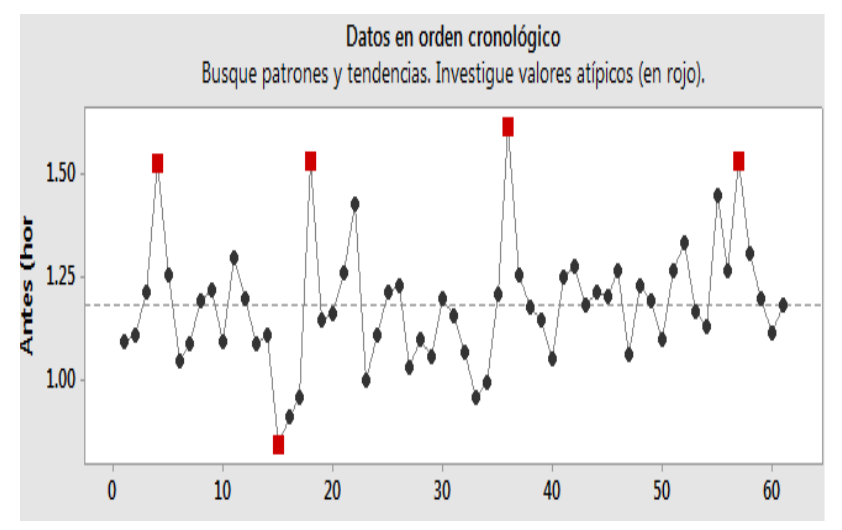

Gráfico 2 Comportamiento de los tiempos de cambio antes de la mejora

Fuente: Elaboración Propia

De acuerdo con las dimensiones consideradas en la variable independiente (SMED), se procedió a realizar el cálculo como se indica en la tabla 9.

\begin{tabular}{|c|c|c|c|}
\hline Variable & Dimen. & Fórmula & Res. \\
\hline \multirow{4}{*}{ SMED } & & $\mathrm{TCC}=$ & $1.18 \mathrm{hrs}$ \\
\hline & $\begin{array}{l}\text { Lambio de } \\
\text { modelo }\end{array}$ & Tiempo consumido $p$ & \multirow{2}{*}{$\begin{array}{l}\frac{1.18 h r s}{11 h r s}= \\
10.72 \%\end{array}$} \\
\hline & & Tiempo disponible & \\
\hline & Disponibilidad & $D=\frac{\text { Tiempo operativo }}{\text { Tiempo disponible }}$ & $\frac{9.82 \mathrm{hrs}}{11 \mathrm{hrs}}=$ \\
\hline
\end{tabular}

Tabla 9 Matriz de operacionalización de variable independiente
En el grafico 3 se observa que el 100\% de tiempo disponible se utiliza un $10.72 \%$ para los cambios de modelo, la disponibilidad de la máquina CHS es del $89.27 \%$, reflejando la necesidad de mejora en la línea de producción.

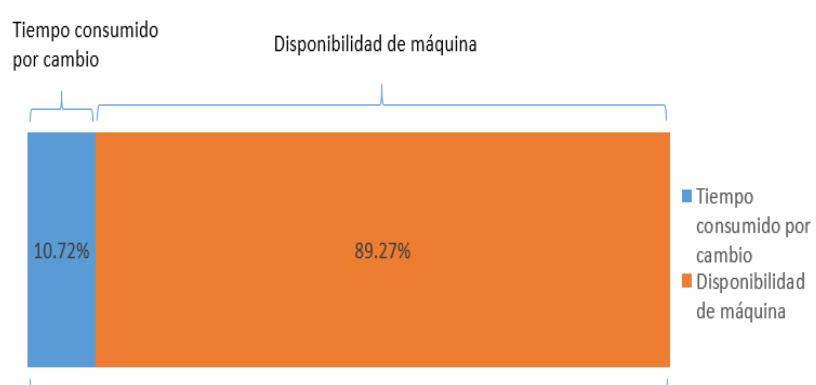

11 horas disponibles

Gráfico 3 Tiempo consumido por cambio de modelo y disponibilidad de máquina antes de la mejora

Con respecto a la variable dependiente (productividad) antes de la mejora, se realizaron las operaciones de las dimensiones eficiencia y eficacia, los resultados obtenidos se muestran en la tabla 10.

Tabla 10 Matriz de operacionalización de variable dependiente

Uno de los beneficios posteriores a la implementación de la herramienta SMED en la línea CHS, fue la disminución de los tiempos por cambio de modelo como se visualiza en el grafico 4 presentando un tiempo promedio de 27.6 minutos (0.46 horas).

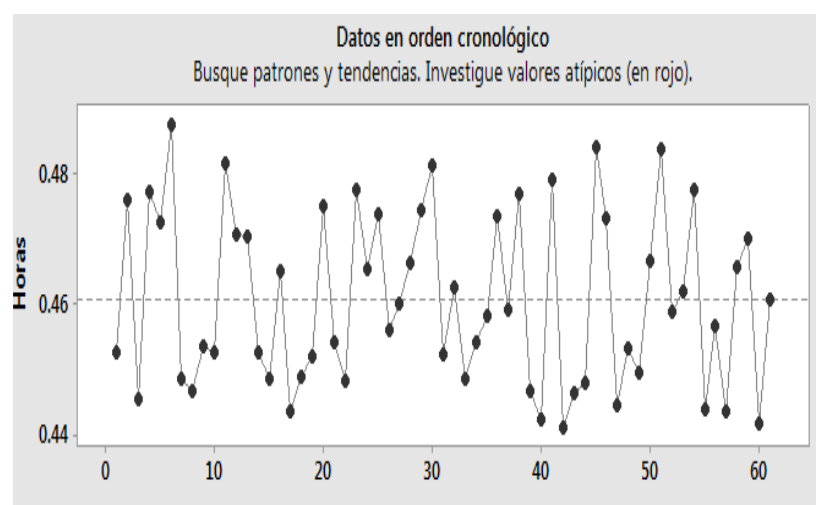

Gráfico 4 Comportamiento de los tiempos de cambio después de la mejora

Fuente: Elaboración Propia

Después de realizar las actividades correspondientes para la implementación del SMED en línea CHS, se procedió a realizar el cálculo como se indica en la tabla 11 referentes a las dimensiones consideradas en la variable independiente (SMED) después de la mejora.

MARTINEZ-HERNANDEZ, Julio Cesar, CRUZ-SOLIS, Edgar Jesús, GARRIDO-ROSADO, Rafael y SANTIAGO-ESCUDERO, Anselmo. Reducción de tiempos de espera en el cambio de modelo mediante la aplicación de la herramienta SMED, un caso de estudio. Revista de Ingeniería Industrial. 2019 


\begin{tabular}{|c|c|c|c|}
\hline Variable & Dimen. & Fórmula & Res. \\
\hline \multirow{4}{*}{ SMED } & Cambio & $\mathrm{TCC}=$ & \multirow{2}{*}{$\frac{0.46 h r s}{11 h r s}=$} \\
\hline & de & Tiempo consumido por camb & \\
\hline & $n$ & Tiemno onerativo & $10.54 \mathrm{hrs}$ \\
\hline & $\begin{array}{l}\text { Disponib1 } \\
\text { lidad. }\end{array}$ & $D M=\frac{T \text { Tiempo disponible }}{\text { Tispon }}$ & $\begin{array}{l}\overline{11 \mathrm{hrs}}= \\
95.81 \%\end{array}$ \\
\hline
\end{tabular}

Tabla 11 Matriz de operacionalización de variable independiente

Fuente: Elaboración Propia

En el grafico 5 se observa que el $100 \%$ de tiempo disponible se redujo a un $4.18 \%$ para los cambios de modelo, la disponibilidad de la máquina CHS se incrementó y alcanzo un $95.81 \%$, reflejando impactos positivos después del proceso de implementación.

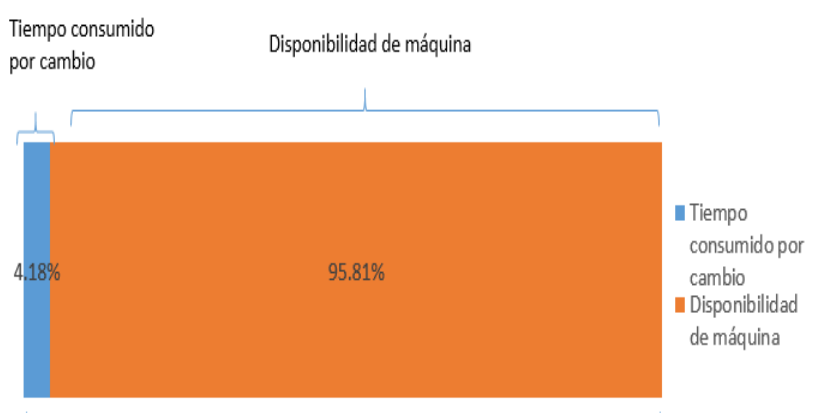

11 horas disponibles

Gráfico 5 Tiempo consumido por cambio de modelo y disponibilidad de máquina después de la mejora Fuente: Elaboración Propia

En la tabla 12 se realizaron las operaciones de las dimensiones eficiencia y eficacia, de acuerdo con la variable dependiente (productividad) después de la mejora.

\begin{tabular}{|c|c|c|c|}
\hline Variable & Dimen. & Fórmula & Res. \\
\hline \multirow{2}{*}{ Productividad } & Eficiencia & $\begin{array}{l}\text { Eficiencia } \\
=\frac{\text { Horas máquinas realizdas }}{\text { Horas máquinas programada }}\end{array}$ & $\begin{array}{l}\frac{10.54 h r s}{11 h r s} \\
=0.95\end{array}$ \\
\hline & Eficacia & $\begin{array}{l}\text { Eficacia } \\
=\frac{\text { Producción real }}{\text { Producción programada }}\end{array}$ & $\begin{array}{l}\frac{14385 \text { libra }}{16000 \text { libras }} \\
=0.89\end{array}$ \\
\hline
\end{tabular}

Tabla 12 Matriz de operacionalización de variable dependiente

Por último, se realizó el comparativo mostrado en el grafico 6, este muestra el comportamiento de la productividad antes y después de la aplicación de la técnica SMED para un periodo de análisis de 30 días, es decir 60 turnos, así mismo la productividad registrada al inicio de este proyecto fue de $68 \%$ (en color azul), posteriormente como resultado de la aplicación sistemática de la metodología desarrollada, se logró incrementar al $86 \%$ (en color rojo).

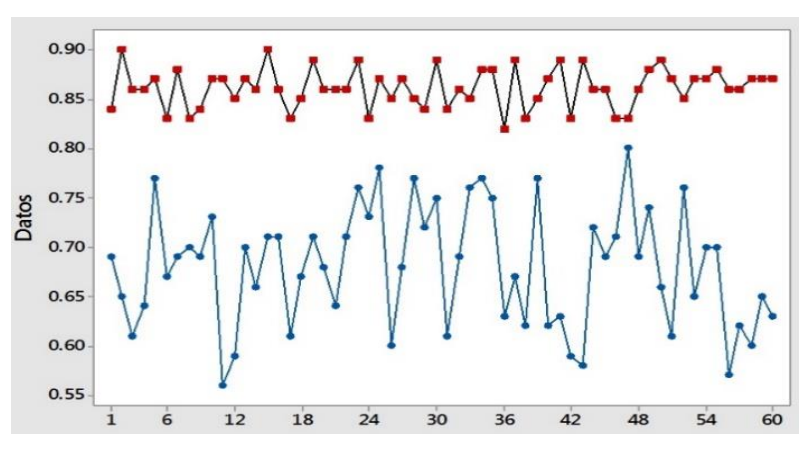

Gráfico 6 Comportamiento de la productividad antes y después de la mejora

\section{Agradecimiento}

A la carrera de Ingeniería Industrial del Instituto Tecnológico Superior de Huauchinango por el apoyo y las facilidades en el desarrollo del proyecto, así mismo, este artículo fue realizado como producto de la colaboración interinstitucional entre los cuerpos académicos de Tecnología Aplicada del ITSH y Ciencias de la Ingeniería del ITSSNP.

\section{Conclusiones}

La mayoría de las organizaciones, de acuerdo con su operación diaria, no disponen de la información detallada necesaria para determinar las pérdidas que se producen durante sus procesos, en consecuencia, no pueden determinar la causa raíz de sus problemas (Gutiérrez, 2010). El éxito del proyecto dependió de la correcta ejecución de la metodología propuesta, tal y como recomienda Socconini (2010), así mimo se utilizaron técnicas estadísticas y Lean Manufacturing para el sustento y cuantificación de las problemáticas presentes en la línea CHS, en cuanto a instrumentos, la toma y registro de tiempos con cronometro para los cambios de modelo fue clave para el análisis de las actividades críticas. A través de la implementación de la herramienta SMED en la línea CHS, se obtuvo un impacto monetario significativo, puesto que las 1500 libras retrasadas por turno corresponden a \$692.31 USD (\$1384.62 USD por día), gasto que a partir de la aplicación del proyecto dejo de representar una perdida para la empresa y el inicio de una filosofía de trabajo orientada a la reducción de desperdicios y la mejora continua de todos sus procesos. El impacto del proyecto se vio reflejado en la productividad de la línea CHS, puesto que se logró un incremento del $18 \%$, así mismo se redujo en un $23 \%$ las jornadas de trabajo extraordinarias para cumplir con las ordenes de producción diarias. 
La técnica SMED es una herramienta poderosa que si se desarrolla siguiendo las instrucciones y recomendaciones de distintos autores como Socconini (2010), Hernández (2013) y Huerta (2017), puede impactar de manera significativa en la productividad; además de ser una técnica fácil de aplicar, consigue resultados rápidos y positivos, algunos beneficios son: aumento de la capacidad de producción, incremento del número de cambios de modelo, reducción de tiempos de ciclo, disminución de tiempos de entrega, entre otros; no obstante durante el desarrollo se pueden presentar limitantes ya que lejos de una resistencia al cambio existe una disposición a mejorar el flujo productivo de las empresas.

La competitividad del mercado actual obliga a disponer de sistemas flexibles que permitan una adaptación a los cambios constantes, y por lo tanto cada vez tienen más importancia las pequeñas series, que además contribuyen a reducir los niveles de stocks tanto en producto acabado, como en material en curso (Rajadell, 2010). Este proyecto fue producto de la vinculación entre la institución de educación superior y el sector industrial, con la finalidad de tecnificar sus procesos e incrementar su productividad.

\section{Comentarios finales}

Derivado de los beneficios obtenidos a través de la aplicación del proyecto, la empresa sugirió la implementación de la herramienta SMED en la línea de panel, esta presenta elevados tiempos de espera, para trabajos a futuro se recomienda combinar la herramienta SMED con ingeniería de métodos y control estadístico para estandarizar las actividades y tener evidencia del comportamiento del proceso, con la finalidad de presentar soluciones que impacten de manera sistemática en los procesos industriales.

Toda empresa debe optar por estrategias, medidas y filosofías orientadas a la Manufactura esbelta, puesto que es una fuente de mejora y está relacionada con la calidad en el ciclo de la mejora continua, por lo que implementar Lean Manufacturing como una estrategia, no solo conlleva una ejecución, sino también busca tener un impacto en todas las personas involucradas en el proceso, ya sea operativos, directivos, etc., así como incrementar la productividad.

\section{Referencias}

Gonzales, C., Isaac, M., \& Escriba Gutiérrez, M. G. (2019). Propuesta de mejora en el proceso de costura de las PYME del sector exportador de confecciones de prendas de vestir de tejido de punto de algodón aplicando herramientas Lean basadas en celdas de manufactura flexible y sistema Pull.

Gutiérrez Pulido H., de la Vara Salazar R. (2009). Control estadístico de calidad y seis sigmas, Segunda edición, México, editorial McGraw-Hill.

Gutiérrez Pulido H. (2010). Calidad total y productividad, Tercera edición, México, D.F., McGraw-Hill.

Hernández Matías J.C., Vizán Idoipe A. (2013). Lean Manufacturing; conceptos, técnicas e implementación, Madrid, Editorial Escuela de organización industrial.

Huerta V. S. (2017). Análisis y propuesta de mejora en la productividad de una línea de envasado de desodorantes utilizando la metodología SMED. (Tesina de grado). Universidad Nacional Mayor De San Marcos. Lima. Perú.

Rajadell Carreras Manuel, Sánchez García José Luis (2010). Lean Manufacturing: La evidencia de una necesidad, Albasanz, Madrid, Ediciones Díaz de Santos.

RAE (2018). Diccionario de la Real Academia Española. Recuperado de https://dle.rae.es/ $\mathrm{srv} / \mathrm{search} ? \mathrm{~m}=30 \& \mathrm{w}=$ desperdicio.

Roqueme Salazar. E., Suarez Ballesteros L. (2015). Implementación de la Metodología Lean para el mejoramiento del proceso comercial de la pyme Tres60 logística, Bogotá DC, Universidad Militar Nueva Granada.

Socconini L. (2008). Lean Manufacturing: Paso a paso, México; Editorial Norma Ediciones.

Vergara, I. G. P., \& López, J. A. R. (2019). Lean, Seis Sigma y Herramientas Cuantitativas: Una Experiencia Real en el Mejoramiento Productivo de Procesos de la Industria Gráfica en Colombia//Lean, Six Sigma and Quantitative Tools: A Real Experience in the Productive Improvement of Processes of th. Revista de Métodos Cuantitativos para la Economía y la Empresa.

MARTINEZ-HERNANDEZ, Julio Cesar, CRUZ-SOLIS, Edgar Jesús, GARRIDO-ROSADO, Rafael y SANTIAGO-ESCUDERO, Anselmo. Reducción de tiempos de espera en el cambio de modelo mediante la aplicación de la herramienta SMED, un caso de estudio. Revista de Ingeniería Industrial. 2019 